\title{
A COUNTEREXAMPLE CONCERNING ALMOST CONTINUOUS FUNCTIONS ${ }^{1}$
}

\author{
LARRY L. HERRINGTON, W. N. HUNSAKER, W. F. LINDGREN, \\ S. A. NAIMPALLY ${ }^{2}$
}

\begin{abstract}
An example is constructed of a function which is almost continuous in the sense of Singal and Singal but not in the sense of Stallings.
\end{abstract}

Let $X$ be the set of real numbers with the topology $\tau$ consisting of the usual open sets together with the sets of the form $U \cap D$, where $U$ is an open set in the usual topology and $D$ the set of all irrational numbers. Let $f:[0,1] \rightarrow(X, \tau)$ be defined by $f(x)=x$. Then $f$ is almost continuous in the sense of Singal and Singal (and also in the sense of Husain ${ }^{3}$ ). Since the only continuous functions on $[0,1] \rightarrow(X, \tau)$ are the constant functions (Steen and Seeback [3, p. 89]), $f$ is not almost continuous in the sense of Stallings. This answers an open problem recently posed by Long and Carnahan [2].

\section{REFERENCES}

1. H. Blumberg, New properties of all real functions, Trans. Amer. Math. Soc. 24 (1922), 113-128.

2. P. E. Long and D. A. Carnahan, Comparing almost continuous functions, Proc. Amer. Math. Soc. 38 (1973), 413-418.

3. L. A. Steen and J. A. Seeback, Jr., Counterexamples in topology, Holt, Rinehart and Winston, New York, 1970. MR 42 \#1040.

University of ArKansas, Fayetteville, Arkansas 72701

SOUthern Illinois University, CaRbondale, Illinois 62901

Slippery Rock State College, Slippery Rock, Pennsylvania 16057

Lakehead University, Thunder Bay, Ontario P7B 5E1, Canada

Received by the editors July 12, 1973.

AMS (MOS) subject classifications (1970). Primary 54C10.

Key words and phrases. Almost continuous functions, noncontinuous functions.

1 A manuscript approximating this one in content, written by the first-named author, was received by the Society July 8,1973 . The present manuscript, coauthored by the last three authors, was received by the Society July 12, 1973. This combined manuscript is printed with the approval of all authors-Ed.

${ }_{2}^{2}$ This research was partially supported by an operating grant from NRC (Canada).

${ }^{3}$ An almost continuous function in the sense of Husain was earlier defined by Blumberg [1].

(c) American Mathematical Society 1974 